\title{
Positive solutions of third-order nonlocal boundary value problems at resonance
}

Hai-E Zhang ${ }^{1 *}$ and Jian-Ping Sun ${ }^{2}$

${ }^{*}$ Correspondence:

haiezhang@126.com

'Department of Basic Teaching,

Tangshan College, Tangshan, Hebei

063000, People's Republic of China

Full list of author information is

available at the end of the article

\begin{abstract}
In this paper, we investigate the existence of positive solutions for a class of third-order nonlocal boundary value problems at resonance. Our results are based on the Leggett-Williams norm-type theorem, which is due to O'Regan and Zima. An example is also included to illustrate the main results.

MSC: 34B10; 34B15
\end{abstract}

Keywords: third-order; nonlocal; at resonance; positive solution

\section{Introduction}

This paper is devoted to the existence of positive solutions for the following third-order nonlocal boundary value problem (BVP for short):

$$
\left\{\begin{array}{l}
x^{\prime \prime \prime}(t)+f(t, x(t))=0, \quad 0<t<1, \\
x(0)=\sum_{i=1}^{m-2} \alpha_{i} x\left(\xi_{i}\right), \quad x^{\prime}(0)=x^{\prime}(1)=0,
\end{array}\right.
$$

where $0<\xi_{1}<\xi_{2}<\cdots<\xi_{m-2}<1, \alpha_{i} \in R^{+}(i=1,2, \ldots, m-2)$ and $\sum_{i=1}^{m-2} \alpha_{i}=1$. The problem (1.1) happens to be at resonance in the sense that the associated linear homogeneous BVP

$$
\left\{\begin{array}{l}
-x^{\prime \prime \prime}(t)=0, \quad 0<t<1, \\
x(0)=\sum_{i=1}^{m-2} \alpha_{i} x\left(\xi_{i}\right), \quad x^{\prime}(0)=x^{\prime}(1)=0,
\end{array}\right.
$$

has nontrivial solutions. Clearly, the resonant condition is $\sum_{i=1}^{m-2} \alpha_{i}=1$. Third-order differential equations arise in a variety of different areas of applied mathematics and physics, e.g., in the deflection of a curved beam having a constant or varying cross section, a threelayer beam, electromagnetic waves or gravity-driven flows and so on [1].

Recently, the existence of positive solutions for third-order two-point or multi-point BVPs has received considerable attention; we mention a few works: [2-11] and the references therein. However, all of the papers on third-order BVPs focused their attention on the positive solutions with non-resonance cases. It is well known that the problem of the existence of positive solutions to BVPs is very difficult when the resonant case is considered. Only few papers deal with the existence of positive solutions to BVPs at resonance, and just to second-order BVPs [12-15]. It is worth mentioning that Infante and Zima [13]

\section{照 Springer}

(c) 2012 Zhang and Sun; licensee Springer. This is an Open Access article distributed under the terms of the Creative Commons Attribution License (http://creativecommons.org/licenses/by/2.0), which permits unrestricted use, distribution, and reproduction in any medium, provided the original work is properly cited. 
studied the existence of positive solutions for the second-order $m$-point BVP

$$
\left\{\begin{array}{l}
-x^{\prime \prime}(t)+f(t, x(t))=0, \quad 0<t<1, \\
x^{\prime}(0)=0, \quad x(1)=\sum_{i=1}^{m-2} \alpha_{i} x\left(\eta_{i}\right)
\end{array}\right.
$$

by means of the Leggett-Williams norm-type theorem due to O'Regan and Zima [16], where $0<\eta_{1}<\eta_{2}<\cdots<\eta_{m-2}<1, \alpha_{i} \in R^{+}(i=1,2, \ldots, m-2)$ and $\sum_{i=1}^{m-2} \alpha_{i}=1$.

However, third-order or higher-order derivatives do not have the convexity; to the best of our knowledge, no results are available for the existence of positive solutions for thirdorder or higher- order BVPs at resonance. The main purpose of this paper is to fill the gap in this area. Motivated greatly by the above-mentioned excellent works, in this paper we will investigate the third-order nonlocal BVP (1.1) at resonance, where $0<\xi_{1}<\xi_{2}<$ $\cdots<\xi_{m-2}<1, \alpha_{i} \in R^{+}(i=1,2, \ldots, m-2)$ and $\sum_{i=1}^{m-2} \alpha_{i}=1$. Some new existence results of at least one positive solution are established by applying the Leggett-Williams norm-type theorem due to O'Regan and Zima [16]. An example is also included to illustrate the main results.

\section{Some definitions and a fixed point theorem}

For the convenience of the reader, we present here the necessary definitions and a new fixed point theorem due to O'Regan and Zima.

Definition 2.1 Let $X$ and $Z$ be real Banach spaces. A linear operator $L: \operatorname{dom} L \subset X \rightarrow Z$ is called a Fredholm operator if the following two conditions hold:

(i) $\operatorname{Ker} L$ has a finite dimension, and

(ii) $\operatorname{Im} L$ is closed and has a finite codimension.

Throughout the paper, we will assume that

$1^{\circ} L$ is a Fredholm operator of index zero, that is, $\operatorname{Im} L$ is closed and $\operatorname{dim} \operatorname{Ker} L=$ codim $\operatorname{Im} L<\infty$.

From Definition 2.1, it follows that there exist continuous projectors $P: X \rightarrow X$ and $Q: Z \rightarrow Z$ such that

$$
\operatorname{Im} P=\operatorname{Ker} L ; \quad \operatorname{Ker} Q=\operatorname{Im} L ; \quad X=\operatorname{Ker} L \oplus \operatorname{Ker} P ; \quad Z=\operatorname{Im} L \oplus \operatorname{Im} Q
$$

and that the isomorphism

$$
\left.L\right|_{\operatorname{dom} L \cap \operatorname{Ker} P}: \operatorname{dom} L \cap \operatorname{Ker} P \rightarrow \operatorname{Im} L
$$

is invertible. We denote the inverse of $\left.L\right|_{\operatorname{dom} L \cap \operatorname{Ker} P}$ by $K_{P}: \operatorname{Im} L \rightarrow \operatorname{dom} L \cap \operatorname{Ker} P$. The generalized inverse of $L$ denoted by $K_{P, Q}: Z \rightarrow \operatorname{dom} L \cap \operatorname{Ker} P$ is defined by $K_{P, Q}=K_{P}(I-Q)$. Moreover, since $\operatorname{dim} \operatorname{Im} Q=\operatorname{codim} \operatorname{Im} L$, there exists an isomorphism $J: \operatorname{Im} Q \rightarrow \operatorname{Ker} L$. Consider a nonlinear operator $N: X \rightarrow Z$. It is known (see $[17,18]$ ) that the coincidence equation $L x=N x$ is equivalent to

$$
x=(P+J Q N) x+K_{P, Q} N x .
$$


Definition 2.2 Let $X$ be a real Banach space. A nonempty closed convex set $P$ is said to be a cone provided that

(1) $\lambda x \in P$ for all $x \in P$ and $\lambda \geq 0$, and

(2) $x,-x \in P$ implies $x=\theta$.

Note that every cone $P \subset X$ induces a partial order $\leq$ in $X$ by defining $x \leq y$ if and only if $y-x \in P$. The following property is valid for every cone in a Banach space.

Lemma 2.3 ([19]) Let $P$ be a cone in $X$. Then for every $u \in P \backslash\{\theta\}$, there exists a positive number $\sigma(u)$ such that $\|x+u\| \geq \sigma(u)\|x\|$ for all $x \in P$.

Let $\gamma: X \rightarrow P$ be a retraction, that is, a continuous mapping such that $\gamma(x)=x$ for all $x \in P$. Set

$$
\Psi=P+J Q N+K_{P, Q} N
$$

and

$$
\Psi_{\gamma}=\Psi \circ \gamma .
$$

Our main results are based on the following theorem due to O'Regan and Zima.

Theorem 2.4 ([16]) Let $C$ be a cone in $X$ and let $\Omega_{1}, \Omega_{2}$ be open bounded subsets of $X$ with $\bar{\Omega}_{1} \subset \Omega_{2}$ and $C \cap\left(\bar{\Omega}_{2} \backslash \Omega_{1}\right) \neq \theta$. Assume that $1^{\circ}$ is satisfied and if the following assumptions hold:

(H1) $Q N: X \rightarrow Z$ is continuous and bounded, and $K_{P, Q}: X \rightarrow X$ is compact on every bounded subset of $X$;

(H2) $L x \neq \lambda N x$ for all $x \in C \cap \partial \Omega_{2} \cap \operatorname{dom} L$ and $\lambda \in(0,1)$;

(H3) $\gamma$ maps subsets of $\bar{\Omega}_{2}$ into bounded subsets of $C$;

(H4) $d_{B}\left(\left.[I-(P+J Q N) \gamma]\right|_{\operatorname{Ker} L}, \operatorname{Ker} L \cap \Omega_{2}, \theta\right) \neq 0$, where $d_{B}$ stands for the Brouwer degree;

(H5) there exists $u_{0} \in C \backslash\{\theta\}$ such that $\|x\| \leq \sigma\left(u_{0}\right)\|\Psi(x)\|$ for $x \in C\left(u_{0}\right) \cap \partial \Omega_{1}$, where $C\left(u_{0}\right)=\left\{x \in C: \mu u_{0} \leq x\right.$ for some $\left.\mu>0\right\}$ and $\sigma\left(u_{0}\right)$ is such that $\left\|x+u_{0}\right\| \geq \sigma\left(u_{0}\right)\|x\|$ for all $x \in C$;

(H6) $(P+J Q N) \gamma\left(\partial \Omega_{2}\right) \subset C$;

(H7) $\Psi_{\gamma}\left(\bar{\Omega}_{2} \backslash \Omega_{1}\right) \subset C$,

then the equation $L x=N x$ has a solution in the set $C \cap\left(\bar{\Omega}_{2} \backslash \Omega_{1}\right)$.

\section{Main results}

For simplicity of notation, we set

$$
h_{i}(s)= \begin{cases}\frac{2 \xi_{i} s-\xi_{i}^{2} s-s^{2}}{2}, & 0 \leq s \leq \xi_{i}, \\ \frac{\xi_{i}^{2}(1-s)}{2}, & \xi_{i} \leq s \leq 1,\end{cases}
$$

where $i=1,2, \ldots, m-2$, and

$$
G(t, s)= \begin{cases}\frac{(1-s)\left(s^{2}-2 s+3 t^{2}\right)}{6}-\frac{(t-s)^{2}}{2}+\frac{4 t^{3}-6 t^{2}+23}{2 \sum_{i=1}^{m-2} \alpha_{i}\left(3 \xi_{i}^{2}-2 \xi_{i}^{3}\right)} \sum_{i=1}^{m-2} \alpha_{i} h_{i}(s), & 0 \leq s \leq t \leq 1, \\ \frac{(1-s)\left(s^{2}-2 s+3 t^{2}\right)}{6}+\frac{4 t^{3}-6 t^{2}+23}{2 \sum_{i=1}^{m-2} \alpha_{i}\left(3 \xi_{i}^{2}-2 \xi_{i}^{3}\right)} \sum_{i=1}^{m-2} \alpha_{i} h_{i}(s), & 0 \leq t \leq s \leq 1 .\end{cases}
$$


It is easy to check that $G(t, s) \geq 0, t, s \in[0,1]$, and since $0 \leq h_{i}(s) \leq \frac{1}{2} s(1-s)$, we get

$$
1-\frac{12 \delta}{\sum_{i=1}^{m-2} \alpha_{i}\left(3 \xi_{i}^{2}-2 \xi_{i}^{3}\right)} \sum_{i=1}^{m-2} \alpha_{i} h_{i}(s) \geq 0, \quad s \in[0,1]
$$

for every $\delta \in\left(0, \frac{2 \xi_{1}^{2}\left(3-2 \xi_{1}\right)}{3}\right]$. We also let $\delta:=\min \left\{\frac{2 \xi_{1}^{2}\left(3-2 \xi_{1}\right)}{3}, \frac{1}{\max _{t, s \in[0,1]} G(t, s)}, 1\right\}$.

We can now state our result on the existence of a positive solution for the BVP (1.1).

Theorem 3.1 Assume that $f:[0,1] \times[0,+\infty) \rightarrow R$ is continuous and

(1) there exists a constant $M \in(0, \infty)$ such that $f(t, x)>-\delta x$ for all $(t, x) \in[0,1] \times[0, M]$;

(2) there exist $r \in(0, M), t_{0} \in[0,1], a \in(0,1], b \in(0,1)$ and continuous functions

$g:[0,1] \rightarrow[0, \infty), h:(0, r] \rightarrow[0, \infty)$ such that $f(t, x) \geq g(t) h(x)$ for

$(t, x) \in[0,1] \times(0, r], \frac{h(x)}{x^{a}}$ is non-increasing on $(0, r]$ with

$$
\frac{h(r)}{(r)^{a}} \int_{0}^{1} G\left(t_{0}, s\right) g(s) d s \geq \frac{1-b}{b^{a}} .
$$

(3) $f_{\infty}=\liminf _{x \rightarrow+\infty} \min _{t \in[0,1]} \frac{f(t, x)}{x}<0$.

Then the resonant $B V P(1.1)$ has at least one positive solution on $[0,1]$.

Proof Consider the Banach spaces $X=Z=C[0,1]$ with $\|x\|=\max _{t \in[0,1]}|x(t)|$.

Let $L: \operatorname{dom} L \subset X \rightarrow Z$ and $N: X \rightarrow Z$ with

$$
\operatorname{dom} L=\left\{x \in X: x^{\prime \prime \prime} \in C[0,1], x(0)=\sum_{i=1}^{m-2} \alpha_{i} x\left(\xi_{i}\right), x^{\prime}(0)=x^{\prime}(1)=0\right\}
$$

be given by $(L x)(t)=-x^{\prime \prime \prime}(t)$ and $(N x)(t)=f(t, x(t))$ for $t \in[0,1]$. Then

$\operatorname{Ker} L=\{x \in \operatorname{dom} L: x(t) \equiv c$ on $[0,1]\}$

and

$$
\operatorname{Im} L=\left\{y \in Z: \sum_{i=1}^{m-2} \alpha_{i} \int_{0}^{1} h_{i}(s) y(s) d s=0\right\} .
$$

Clearly, $\operatorname{dim} \operatorname{Ker} L=1$ and $\operatorname{Im} L$ is closed. It follows from $Z_{1}=Z \backslash \operatorname{Im} L$ that

$$
Z_{1}=\left\{y_{1} \in Z: y_{1}=\frac{12}{2 \sum_{i=1}^{m-2} \alpha_{i}\left(3 \xi_{i}^{2}-2 \xi_{i}^{3}\right)} \sum_{i=1}^{m-2} \alpha_{i} \int_{0}^{1} h_{i}(s) y(s) d s, y \in Z\right\} .
$$

In fact, for each $y \in Z$, we have

$$
\begin{aligned}
& \sum_{i=1}^{m-2} \alpha_{i} \int_{0}^{1} h_{i}(s)\left(y(s)-y_{1}(s)\right) d s \\
& \quad=\left(1-\frac{12}{\sum_{i=1}^{m-2} \alpha_{i}\left(3 \xi_{i}^{2}-2 \xi_{i}^{3}\right)} \sum_{i=1}^{m-2} \alpha_{i} \int_{0}^{1} h_{i}(s) d s\right) \sum_{i=1}^{m-2} \alpha_{i} \int_{0}^{1} h_{i}(s) y(s) d s=0,
\end{aligned}
$$


which shows that $y-y_{1} \in \operatorname{Im} L$, which together with $Z_{1} \cap \operatorname{Im} L=\{\theta\}$ implies that $Z=Z_{1} \oplus$ $\operatorname{Im} L$. Note that $\operatorname{dim} Z_{1}=1$ and thus $\operatorname{codim} \operatorname{Im} L=1$. Therefore, $L$ is a Fredholm operator of index zero.

Next, define the projections $P: X \rightarrow X$ by

$$
P x=\int_{0}^{1} x(s) d s
$$

and $Q: Z \rightarrow Z$ by

$$
Q y=\frac{12}{\sum_{i=1}^{m-2} \alpha_{i}\left(3 \xi_{i}^{2}-2 \xi_{i}^{3}\right)} \sum_{i=1}^{m-2} \alpha_{i} \int_{0}^{1} h_{i}(s) y(s) d s .
$$

Clearly, $\operatorname{Im} P=\operatorname{Ker} L, \operatorname{Ker} Q=\operatorname{Im} L$ and $\operatorname{Ker} L=\left\{x \in X: \int_{0}^{1} x(s) d s=0\right\}$. Note that for $y \in$ $\operatorname{Im} L$, the inverse $K_{P}$ of $L_{P}$ is given by

$$
\left(K_{P} y\right)(t)=\int_{0}^{1} k(t, s) y(s) d s
$$

where

$$
k(t, s)= \begin{cases}\frac{(1-s)\left(s^{2}-2 s+3 t^{2}\right)}{6}-\frac{(t-s)^{2}}{2}, & 0 \leq s \leq t \leq 1, \\ \frac{(1-s)\left(s^{2}-2 s+3 t^{2}\right)}{6}, & 0 \leq t \leq s \leq 1\end{cases}
$$

Considering that $f$ can be extended continuously to $[0,1] \times R$, it is easy to check that $Q N: X \rightarrow Z$ is continuous and bounded, and $K_{P, Q} N: X \rightarrow X$ is compact on every bounded subset of $X$, which ensures that (H1) of Theorem 2.4 is fulfilled.

Define the cone of nonnegative functions

$$
C=\{x \in X: x(t) \geq 0, \text { on }[0,1]\} .
$$

Let

$$
\Omega_{1}=\{x \in X: b\|x\|<|x(t)|<r, t \in[0,1]\}
$$

and

$$
\Omega_{2}=\{x \in X:\|x\|<M\} .
$$

Clearly, $\Omega_{1}$ and $\Omega_{2}$ are bounded and open sets and

$$
\bar{\Omega}_{1}=\{x \in X: b\|x\| \leq|x(t)| \leq r, t \in[0,1]\} \subset \Omega_{2} .
$$

Moreover, $C \cap\left(\bar{\Omega}_{2} \backslash \Omega_{1}\right) \neq \theta$. Let $J=I$ and $(\gamma x)(t)=|x(t)|$ for $x \in X$. Then $\gamma$ is a retraction and maps subsets of $\bar{\Omega}_{2}$ into bounded subsets of $C$, which means that (H3) of Theorem 2.4 holds.

Let $0<r^{\prime}=\min \left\{r_{1}, r_{2}\right\}$, where $r_{1}$ and $r_{2}$ will be defined in the following proof. 
Suppose that there exist $x_{0} \in C \cap \partial \Omega_{2} \cap \operatorname{dom} L$ and $\lambda_{0} \in(0,1)$ such that $L x_{0}=\lambda_{0} N x_{0}$. Then

$$
x_{0}^{\prime \prime \prime}(t)+\lambda_{0} f\left(t, x_{0}(t)\right)=0
$$

Let $x_{0}\left(t_{1}\right)=\left\|x_{0}\right\|=\max _{t \in[0,1]}\left|x_{0}(t)\right|=M$. Now, we verify that $t_{1} \neq 0$ and $t_{1} \neq 1$.

First, we show $t_{1} \neq 0$. Suppose, on the contrary, that $x_{0}(t)$ achieves maximum value $M$ only at $t=0$. Then $x_{0}(0)=\sum_{i=1}^{m-2} \alpha_{i} x_{0}\left(\xi_{i}\right)$ in combination with $\sum_{i=1}^{m-2} \alpha_{i}=1$ yields that $\max _{1 \leq i \leq m-2}\left\{x_{0}\left(\xi_{i}\right)\right\} \geq M$, which is a contradiction.

Next, we show $t_{1} \neq 1$. It follows from $x_{0}^{\prime}(0)=x_{0}^{\prime}(1)=0$ that there is a constant $\eta \in(0,1)$ such that $x_{0}^{\prime \prime}(\eta)=0$, and thus $x_{0}^{\prime \prime}(t)=-\lambda_{0} \int_{\eta}^{t} f\left(s, x_{0}(s)\right) d s$. By the condition (3) we have, for $x \geq r^{\prime}\left(M \geq r^{\prime}>r>0\right)$, there exists $\sigma \in(0, \delta)$ such that

$$
f(t, x) \leq-\sigma x, \quad \forall t \in[0,1]
$$

Suppose, on the contrary, that $x_{0}(1)=M$. The step is divided into two cases:

Case 1. Assume that $x_{0}(t)>0$ on $[\eta, 1]$. Let $r_{1}=\min _{t \in[\eta, 1]} x_{0}(t)$. Then (3.1) yields

$$
x_{0}^{\prime \prime}(1)=-\lambda_{0} \int_{\eta}^{1} f\left(s, x_{0}(s)\right) d s \geq \lambda_{0} \sigma \int_{\eta}^{1} x_{0}(s) d s>0,
$$

which implies $x_{0}^{\prime}(t)$ is increasing close to 1 . This together with $x_{0}^{\prime}(1)=0$ induces $x_{0}^{\prime}(t)<0$ ( $t$ close to 1 ), that is, $x_{0}(t)$ is decreasing close to 1 , which contradicts $x_{0}(1)=M$.

Case 2. Assume that $x_{0}(t)$ has zero points on $[\eta, 1]$; we may choose $\eta^{\prime}$ nearest to 1 with $x_{0}\left(\eta^{\prime}\right)=0$. Then there is a constant $\eta \in\left(\eta^{\prime}, 1\right)$ such that $x_{0}^{\prime \prime}(\eta)=0$. Similar to the above arguments, we easily get a contradiction too.

Hence, we can choose $t_{1} \in(0,1)$ so that $x_{0}\left(t_{1}\right)=M$. This gives $x_{0}^{\prime}\left(t_{1}\right)=0$ and $x_{0}^{\prime \prime}\left(t_{1}\right) \leq 0$. By $x_{0} \in \operatorname{dom} L$, we know $x_{0}(t) \geq 0$ and $x_{0}^{\prime}(0)=0$. Similarly, we also divide the part of the proof into two cases.

Case 1. If $x_{0}(t)>0$ on $\left[0, t_{1}\right]$, then there is a constant $\eta \in\left(0, t_{1}\right)$ such that $x_{0}^{\prime \prime}(\eta)=0$. Thus we have

$$
x_{0}^{\prime \prime}(t)=-\lambda_{0} \int_{\eta}^{t} f\left(s, x_{0}(s)\right) d s
$$

Let $r_{2}=\min _{t \in\left[\eta, t_{1}\right]} x_{0}(t)$. Then it follows from (3.1) that

$$
0 \geq x_{0}^{\prime \prime}\left(t_{1}\right)=-\lambda_{0} \int_{\eta}^{t_{1}} f\left(s, x_{0}(s)\right) d s \geq \lambda_{0} \sigma \int_{\eta}^{t_{1}} x_{0}(s) d s>0,
$$

which is a contradiction.

Case 2. If $x_{0}(t)$ has zero points on $\left[0, t_{1}\right]$, we may choose $\eta^{\prime}$ nearest to $t_{1}$ with $x_{0}\left(\eta^{\prime}\right)=0$. Then there is a constant $\eta \in\left(\eta^{\prime}, t_{1}\right)$ such that $x_{0}^{\prime \prime}(\eta)=0$. Thus we have

$$
x_{0}^{\prime \prime}(t)=-\lambda_{0} \int_{\eta}^{t} f\left(s, x_{0}(s)\right) d s
$$


Let $r_{2}=\min _{t \in\left[\eta, t_{1}\right]} x_{0}(t)$. Then it follows from (3.1) that

$$
0 \geq x_{0}^{\prime \prime}\left(t_{1}\right)=-\lambda_{0} \int_{\eta}^{t_{1}} f\left(s, x_{0}(s)\right) d s \geq \lambda_{0} \sigma \int_{\eta}^{t_{1}} x_{0}(s) d s>0,
$$

which is a contradiction. Therefore, (H2) of Theorem 2.4 holds.

Consider $x \in \operatorname{Ker} L \cap \Omega_{2}$. Then $x(t) \equiv c$ on $[0,1]$. Similar to [13], we define

$$
H(x, \lambda)=x-\lambda|x|-\frac{12 \lambda}{\sum_{i=1}^{m-2} \alpha_{i}\left(3 \xi_{i}^{2}-2 \xi_{i}^{3}\right)} \sum_{i=1}^{m-2} \alpha_{i} \int_{0}^{1} h_{i}(s) f(s,|x|) d s,
$$

$x \in \operatorname{Ker} L \cap \Omega_{2}$ and $\lambda \in[0,1]$.

Suppose $H(x, \lambda)=0$. Then in view of (1), we obtain

$$
\begin{aligned}
c & =\lambda c+\frac{12 \lambda}{\sum_{i=1}^{m-2} \alpha_{i}\left(3 \xi_{i}^{2}-2 \xi_{i}^{3}\right)} \sum_{i=1}^{m-2} \alpha_{i} \int_{0}^{1} h_{i}(s) f(s,|c|) d s \\
& \geq \lambda c-\frac{12 \lambda}{\sum_{i=1}^{m-2} \alpha_{i}\left(3 \xi_{i}^{2}-2 \xi_{i}^{3}\right)} \sum_{i=1}^{m-2} \alpha_{i} \int_{0}^{1} h_{i}(s) \delta|c| d s \geq \lambda|c|(1-\delta) \geq 0 .
\end{aligned}
$$

Hence, $H(x, \lambda)=0$ implies $c \geq 0$. Furthermore, if $H(M, \lambda)=0$, then we have

$$
0 \leq M(1-\lambda)=\frac{12 \lambda}{\sum_{i=1}^{m-2} \alpha_{i}\left(3 \xi_{i}^{2}-2 \xi_{i}^{3}\right)} \sum_{i=1}^{m-2} \alpha_{i} \int_{0}^{1} h_{i}(s) f(s, M) d s,
$$

contradicting (3.1). Thus $H(x, \lambda)=0$ for $x \in \partial \Omega_{2}$ and $\lambda \in[0,1]$. Therefore,

$$
d_{B}\left(H(x, 0), \operatorname{Ker} L \cap \Omega_{2}, \theta\right)=d_{B}\left(H(x, 1), \operatorname{Ker} L \cap \Omega_{2}, \theta\right) .
$$

However,

$$
d_{B}\left(H(x, 0), \operatorname{Ker} L \cap \Omega_{2}, \theta\right)=d_{B}\left(I, \operatorname{Ker} L \cap \Omega_{2}, \theta\right) .
$$

This gives

$$
d_{B}\left(\left.[I-(P+J Q N) \gamma]\right|_{\operatorname{Ker} L}, \operatorname{Ker} L \cap \Omega_{2}, \theta\right)=d_{B}\left(H(x, 1), \operatorname{Ker} L \cap \Omega_{2}, \theta\right) \neq 0,
$$

which shows that (H4) of Theorem 2.4 holds.

Let $x \in \bar{\Omega}_{2} \backslash \Omega_{1}$ and $t \in[0,1]$. Then

$$
\begin{aligned}
& \left(\Psi_{\gamma} x\right)(t) \\
& =\int_{0}^{1}|x(s)| d s+\frac{12}{\sum_{i=1}^{m-2} \alpha_{i}\left(3 \xi_{i}^{2}-2 \xi_{i}^{3}\right)} \sum_{i=1}^{m-2} \alpha_{i} \int_{0}^{1} h_{i}(s) f(s,|x(s)|) d s \\
& \quad+\int_{0}^{1} k(t, s)\left[f(s,|x(s)|)-\frac{12}{\sum_{i=1}^{m-2} \alpha_{i}\left(3 \xi_{i}^{2}-2 \xi_{i}^{3}\right)} \sum_{i=1}^{m-2} \alpha_{i} \int_{0}^{1} h_{i}(\tau) f(\tau,|x(\tau)|) d \tau\right] d s \\
& =\int_{0}^{1}|x(s)| d s+\int_{0}^{1} G(t, s) f(s,|x(s)|) d s .
\end{aligned}
$$


From (1), we know that

$$
\begin{aligned}
\left(\Psi_{\gamma}\right)(t) & \geq \int_{0}^{1}|x(s)| d s-\delta \int_{0}^{1} G(t, s)|x(s)| d s \\
& =\int_{0}^{1}(1-\delta G(t, s))|x(s)| d s \geq 0 .
\end{aligned}
$$

Hence $\Psi_{\gamma}\left(\overline{\Omega_{2}} \backslash \Omega_{1}\right) \subset C$. Moreover, for $x \in \partial \Omega_{2}$, we have

$$
\begin{aligned}
(P+J Q N) \gamma x & =\int_{0}^{1}|x(s)| d s+\frac{12}{\sum_{i=1}^{m-2} \alpha_{i}\left(3 \xi_{i}^{2}-2 \xi_{i}^{3}\right)} \sum_{i=1}^{m-2} \alpha_{i} \int_{0}^{1} h_{i}(s) f(s,|x(s)|) d s \\
& \geq \int_{0}^{1}\left[1-\frac{12 \delta}{2 \sum_{i=1}^{m-2} \alpha_{i}\left(3 \xi_{i}^{2}-2 \xi_{i}^{3}\right)} \sum_{i=1}^{m-2} \alpha_{i} h_{i}(s)\right]|x(s)| d s \geq 0,
\end{aligned}
$$

which shows that $(P+J Q N) \gamma\left(\partial \Omega_{2}\right) \subset P$. These ensure that (H6), (H7) of Theorem 2.4 hold. It remains to show that $(\mathrm{H} 5)$ is satisfied.

Taking $u_{0}(t) \equiv 1$ on $[0,1]$ and $\sigma\left(u_{0}\right)=1$, we confirm that

$$
u_{0} \in C \backslash\{\theta\}, \quad C\left(u_{0}\right)=\{x \in C: x(t)>0 \text { on }[0,1]\}
$$

Let $x \in C\left(u_{0}\right) \cap \partial \Omega_{1}$. Then we have $x(t)>0, t \in[0,1], 0<\|x\|<r$ and $x(t) \geq b\|x\|, t \in[0,1]$. Therefore, in view of (2), for all $x \in C\left(u_{0}\right) \cap \partial \Omega_{1}$, we obtain

$$
\begin{aligned}
(\Psi x)\left(t_{0}\right) & =\int_{0}^{1} x(s) d s+\int_{0}^{1} G\left(t_{0}, s\right) f(s, x(s)) d s \\
& \geq b\|x\|+\int_{0}^{1} G\left(t_{0}, s\right) g(s) h(x(s)) d s \\
& =b\|x\|+\int_{0}^{1} G\left(t_{0}, s\right) g(s) \frac{h(x(s))}{x^{a}(s)} x^{a}(s) d s \\
& \geq b\|x\|+\frac{h(r)}{(r)^{a}} \int_{0}^{1} G\left(t_{0}, s\right) g(s) x^{a}(s) d s \\
& \geq b\|x\|+\frac{h(r)}{(r)^{a}} \int_{0}^{1} G\left(t_{0}, s\right) g(s) b^{a}\|x\|^{a} d s \\
& \geq b\|x\|+(1-b)\|x\|=\|x\| .
\end{aligned}
$$

That is, $\|x\| \leq \sigma\left(u_{0}\right)\|\Psi x\|$ for all $x \in C\left(u_{0}\right) \cap \partial \Omega_{1}$, which shows that (H5) of Theorem 2.4 holds.

Summing up, all the hypotheses of Theorem 2.4 are satisfied. Therefore, the equation $L x=N x$ has a solution $x \in C \cap\left(\bar{\Omega}_{2} \backslash \Omega_{1}\right)$. And so, the resonant BVP (1.1) has at least one positive solution on $[0,1]$.

\section{An example}

Consider the BVP

$$
\left\{\begin{array}{l}
x^{\prime \prime \prime}(t)+f(t, x(t))=0, \quad 0<t<1, \\
x(0)=x\left(\frac{1}{2}\right), \quad x^{\prime}(0)=x^{\prime}(1)=0 .
\end{array}\right.
$$


Here $\alpha_{1}=1, \xi_{1}=\frac{1}{2}, g(t)=-\frac{1}{4}\left(t^{2}-t-1\right), h(x)=\sqrt{x+1}$ and

$$
f(t, x)= \begin{cases}-\frac{1}{4}\left(t^{2}-t-1\right)\left(x^{2}-8 x+15\right) \sqrt{x+1}, & t \in[0,1], x \in[0,2], \\ -\frac{1}{4}\left(t^{2}-t-1\right)\left(2-\frac{1}{2} \sqrt{x-1}\right) \sqrt{x+1}, & t \in[0,1], x \in(2,+\infty) .\end{cases}
$$

By a simple computation, we get

$$
\delta=\frac{1}{3}, \quad \frac{1}{4} \leq g(t) \leq \frac{5}{16}, \quad t \in[0,1], \quad x^{2}-8 x+15 \geq-x, \quad x \in[0,3] .
$$

We may choose $M=3, r=\frac{5}{2}, t_{0}=0, a=1, b=\frac{13}{14}$. It is easy to check

(1) $f(t, x)>-\frac{1}{3} x$ for all $(t, x) \in[0,1] \times[0,3]$;

(2) $f(t, x) \geq g(t) h(x)$ for $(t, x) \in[0,1] \times\left(0, \frac{5}{2}\right], \frac{\sqrt{x+1}}{x}$ is non-increasing on $\left(0, \frac{5}{2}\right]$ with

$$
\frac{h(r)}{(r)^{a}} \int_{0}^{1} G(0, s) g(s) d s \geq \frac{7 \sqrt{14}}{320} \geq \frac{1}{13}=\frac{1-b}{b^{a}}
$$

(3) $f_{\infty}=\liminf _{x \rightarrow+\infty} \min _{t \in[0,1]} \frac{f(t, x)}{x}=-\frac{1}{8}$.

Thus, all the conditions of Theorem 3.1 are satisfied. Then the resonant problem (4.1) has at least one positive solution on $[0,1]$.

\section{Competing interests}

The authors declare that they have no competing interests.

\section{Authors' contributions}

The authors declare that the work was realized in collaboration with same responsibility. All authors read and approved the final manuscript.

\section{Author details}

${ }^{1}$ Department of Basic Teaching, Tangshan College, Tangshan, Hebei 063000, People's Republic of China. ${ }^{2}$ Department of Applied Mathematics, Lanzhou University of Technology, Lanzhou, Gansu 730050, People's Republic of China.

\section{Acknowledgements}

This work is supported by the National Natural Science Foundation of China (10801068) and the Education Scientific Research Foundation of Tangshan College (120186). The authors would like to thank the anonymous referees very much for helpful comments and suggestions which led to the improvement of presentation and quality of the work.

Received: 21 April 2012 Accepted: 3 September 2012 Published: 18 September 2012

\section{References}

1. Gregus, M: Third Order Linear Differential Equations. Math. Appl. Reidel, Dordrecht (1987)

2. Anderson, DR: Green's function for a third-order generalized right focal problem. J. Math. Anal. Appl. 288, 1-14 (2003)

3. Anderson, DR, Davis, JM: Multiple solutions and eigenvalues for three-order right focal boundary value problems. J. Math. Anal. Appl. 267, 135-157 (2002)

4. Du, ZJ, Ge, WG, Zhou, MR: Singular perturbations for third-order nonlinear multi-point boundary value problem. J. Differ. Equ. 218, 69-90 (2005)

5. El-Shahed, M: Positive solutions for nonlinear singular third order boundary value problems. Commun. Nonlinear Sci. Numer. Simul. 14, 424-429 (2009)

6. Li, S: Positive solutions of nonlinear singular third-order two-point boundary value problem. J. Math. Anal. Appl. 323, 413-425 (2006)

7. Liu, ZQ, Debnath, L, Kang, SM: Existence of monotone positive solutions to a third-order two-point generalized right focal boundary value problem. Comput. Math. Appl. 55, 356-367 (2008)

8. Palamides, AP, Smyrlis, G: Positive solutions to a singular third-order three-point boundary value problem with an indefinitely signed Green's function. Nonlinear Anal. 68, 2104-2118 (2008)

9. Sun, JP, Zhang, HE: Existence of solutions to third-order m-point boundary value problems. Electron. J. Differ. Equ. 2008, 125 (2008)

10. Sun, Y: Positive solutions for third-order three-point nonhomogeneous boundary value problems. Appl. Math. Lett 22, 45-51 (2009)

11. Yao, Q: The existence and multiplicity of positive solutions for a third-order three-point boundary value problem Acta Math. Appl. Sin. 19, 117-122 (2003) 
12. Bai, C, Fang, J: Existence of positive solutions for boundary value problems at resonance. J. Math. Anal. Appl. 291, 538-549 (2004)

13. Infante, G, Zima, M: Positive solutions of multi-point boundary value problems at resonance. Nonlinear Anal. 69 2458-2465 (2008)

14. Liang, SQ, Mu, L: Multiplicity of positive solutions for singular three-point boundary value problem at resonance. Nonlinear Anal. 71, 2497-2505 (2009)

15. Yang, L, Shen, CF: On the existence of positive solution for a kind of multi-order boundary value problem at resonance. Nonlinear Anal. 72, 4211-4220 (2010)

16. O'Regan, D, Zima, M: Leggett-Williams norm-type theorems for coincidence. Arch. Math. 87, 233-244 (2006)

17. Mawhin, J: Equivalence theorems for nonlinear operator equations and coincidence degree theory for some mappings in locally convex topological vector spaces. J. Differ. Equ. 72, 4211-4220 (2010)

18. Santanilla, J: Some coincidence theorems in wedges, cones and convex sets. J. Math. Anal. Appl. 105, 357-371 (1985)

19. Petryshyn, $W V$ : On the solvability of $x \in T x+\lambda F x$ in quasinormal cones with $T$ and $F k$-set contractive. Nonlinear Anal. 5, 585-591 (1981)

doi:10.1186/1687-2770-2012-102

Cite this article as: Zhang and Sun: Positive solutions of third-order nonlocal boundary value problems at resonance. Boundary Value Problems 2012 2012:102.

\section{Submit your manuscript to a SpringerOpen ${ }^{\circ}$ journal and benefit from:}

- Convenient online submission

Rigorous peer review

- Immediate publication on acceptance

- Open access: articles freely available online

- High visibility within the field

- Retaining the copyright to your article 